\title{
An On-Line Measurement-Based Admission Control for VBR Video Traffic in Wireless Multimedia Home Networks
}

\author{
Yi-Hsien Tseng ${ }^{1}$, Eric Hsiao-Kuang $\mathrm{Wu}^{2}$, and Gen-Huey Chen ${ }^{1, \star}$ \\ ${ }^{1}$ Department of Computer Science and Information Engineering, \\ National Taiwan University, Taipei, Taiwan \\ ghchen@csie.ntu.edu.tw \\ ${ }^{2}$ Department of Computer Science and Information Engineering, \\ National Central University, Chung-Li, Taiwan
}

\begin{abstract}
Variable bit rate (VBR) video traffic with high data rate is expected to be the largest proportion of traffic carried by future wireless home networks. In order to guarantee quality-of-service (QoS) requirements of such type of VBR video traffic and improve channel utilization, this paper proposes an on-line measurement-based admission control scheme. The proposed scheme is designed based on the property of the aggregate VBR video traffic is lognormal distributed and consists of two main components which are measurement process and admission decision respectively. The measurement process applies a linear Kalman filter to estimate statistical parameters of aggregate VBR video traffic. Then, the estimated statistical parameters are used to calculate the effective bandwidth for admission decision. The proposed scheme is computational efficiently and accurate without much prior information of traffic. Simulation results confirm its accuracy and also show that the proposed scheme performs well for small number of connections as well as large number of connections.
\end{abstract}

\section{Introduction}

Because of ease of installation or relocation, wireless home networks are particularly attractive to ensure required "wire-like" performance for many indoor multimedia applications. Consequently, Variable bit rate (VBR) video traffic is expected to be the largest proportion of traffic carried by future wireless home networks.

In order to guarantee quality-of-service (QoS) requirements of VBR video traffic, an admission control scheme is needed by the network to decide whether a new connection is accepted or rejected. VBR encoding can offer higher picture quality and greater opportunity for statistical multiplexing gains [1] (with the same average bandwidth) than constant bit rate (CBR) encoding. However, an admission control scheme may make decision under uncertain conditions because

\footnotetext{
* Corresponding author.
} 
the required bandwidth of VBR video traffic varies with time. A good admission control scheme for VBR video traffic should improve channel utilization through statistical multiplexing and guarantee QoS at the same time.

Many admission control schemes have been proposed to date (e.g, [2]-7]). In [8], the overview and the three-way taxonomy were provided. They commented that the admission control schemes proposed by [2] and 3] are simple and can achieve high bandwidth efficiency. These two schemes were both based on effective bandwidth and using measurement.

In this paper, we propose an on-line Measurement-based admission control (MBAC) scheme for VBR video traffic. The type of VBR video which we deal with is the full-motion video generated by a Motion Picture Expert Group (MPEG) encoder 9. Several probability distribution functions, such as Gamma, Weibull, lognormal etc., can be used to fit the distribution of MPEG-coded video trace (the sequence of bits per frame in the compressed video traffic).

The proposed scheme consists of two main components which are measurement process and admission decision respectively. A linear Kalman filter [10] is applying in the measurement process to estimate statistical parameters of aggregate VBR video trace. The estimated statistical parameters are used to calculate the effective bandwidth for admission decision. The effective bandwidth is devised from the assumption that the aggregate VBR video trace is lognormal distributed. The proposed scheme can achieve good performances which include channel utilization and QoS guarantee for VBR video traffic. Meanwhile, it is computation effectively and can be adopted to be an on-line MBAC scheme for real-time VBR video traffic. Moreover, the proposed scheme needs not to know much statistical characteristic (only peak rate) of individual VBR video traffic in advance.

The rest of this paper is organized as follows. In Section 2, an on-line MBAC scheme for VBR video traffic is proposed. Simulation and comparison results are shown in Section 3. Finally, this paper concludes with some remarks in Section 4.

\section{A MBAC with Aggregate Effective Bandwidth Estimation}

In this section, we propose a MBAC scheme with aggregate effective bandwidth estimation (MBAC-AEBE) for VBR videos. The MBAC-AEBE is based on the assumption that $\mathrm{VBR}$ video trace is lognormal distributed and consists of two main components which are measurement process and admission decision respectively.

\subsection{Network Model and Aggregate VBR Video Traffic Model}

The network model that we consider is the IEEE 802.15.3 11] wireless home network. The IEEE 802.15.3 MAC protocol uses time division multiple access (TDMA) to allocate channel time among devices, in order to prevent conflicts, and it allocates new channel time for a connection only when enough bandwidth is available. 
The elementary topology unit for the IEEE 802.15.3 MAC layer is a piconet. A piconet contains a number of independent data devices (DEVs) that are allowed to exchange frames directly with each other. The master/slave relationship was adopted for these DEVs; a particular DEV, named piconet coordinator (PNC), acts as the master and the others are slaves. Timing for a piconet is realized by superframes and is shown in Fig. 1.

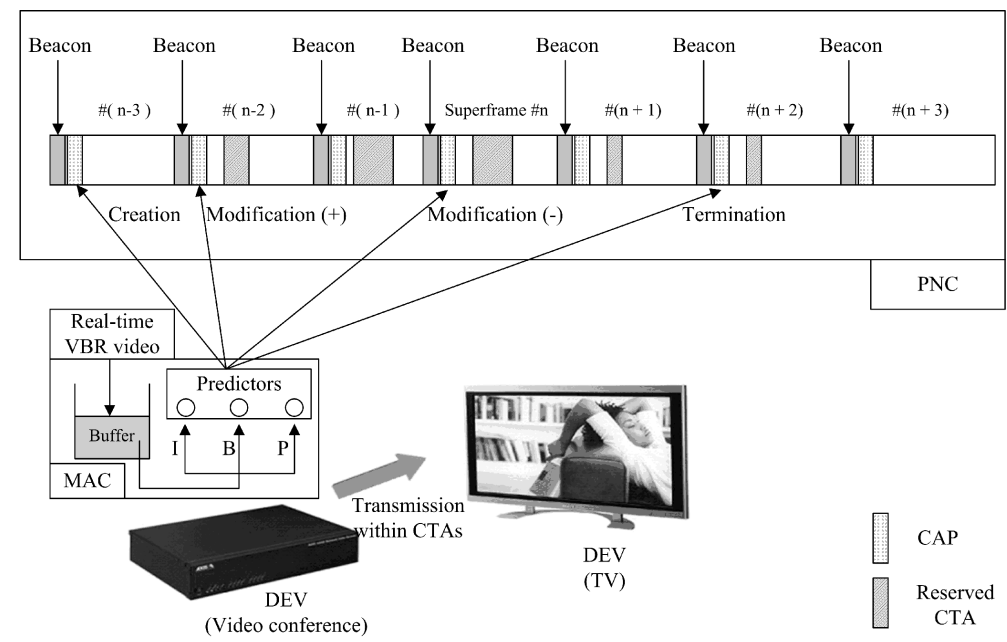

Fig. 1. Timing for a piconet in IEEE 802.15.3 wireless home networks

The MBAC-AEBE is performed in the PNC to determine if a new VBR video traffic is accepted or not. The VBR video traffic that we consider is the fullmotion video traffic generated by a MPEG encoder. MPEG-coded video traces used by this paper can be found in 12. The MPEG introduced three frame types: intra-frame (I), inter-frame (P) and bidirectional-frame (B). These frame types are organized into so called groups of pictures (GoPs). The size of each GoP is measured by the MBAC-AEBE and is used for admission control.

It was shown in 13 , that the distribution of VBR video trace can be fitted well by a lognormal distribution. A random variable $X$ with a lognormal distribution has PDF:

$$
f(x ; \mu, \sigma)=\frac{1}{x \sigma \sqrt{2 \pi}} e^{-(\ln x-\mu)^{2} / 2 \sigma^{2}},
$$

where $\mu$ and $\sigma^{2}$ are the mean and variance of the random variable $Y=\ln (X)$ which is Gaussian (Normal) distributed.

If aggregate GoP sizes are lognormal distributed, they are easy to be transformed into be Gaussian distributed. Then the mean $\mu$ and variance $\sigma^{2}$ of the Gaussian distribution can be estimated by their good unbiased estimators $\bar{X}$ and $S^{2}$ as follows respectively. 


$$
\begin{aligned}
\bar{X} & =\frac{X_{1}+\ldots+X_{n}}{n} . \\
S^{2} & =\frac{\sum_{i=1}^{n}\left(X_{i}-\bar{X}\right)}{n-1} .
\end{aligned}
$$

However, it may not be easy to find good unbiased estimators for other distributions.

\subsection{Measurement Process Using Kalman Filter}

From above discussion, we denote a aggregate GoP trace as a random process $x(n)$ with lognormal distribution. Then, the $y(n)=\ln (x(n))$ is a random process with Gaussian distribution. The measurement process of the MBAC-AEBE adaptively estimates the mean and variance of $y(n)$ in each GoP period. This is done by an optimization scheme based on a linear Kalman filter. The Kalman filter can provide an optimal least-square estimate of the system state for a linear system.

The Kalman filter is a set of mathematical equations that implement a predictor-corrector type estimator that is optimal in the sense that it minimizes the estimated error covariance. The Kalman filter addresses the problem of estimating the state $x \in R^{n}$ of a discrete-time controlled process that is governed by the process equation

$$
x_{k}=A x_{k-1}+w_{k},
$$

with a measurement $z \in R^{m}$ which measurement equation is

$$
z_{k}=B x_{k}+v_{k}
$$

where the $n \times n$ matrix $A$ relates the state at the previous time step $k-1$ to the state at the current step $k$. The $m \times n$ matrix $B$ relates the state $x_{k}$ to the measurement $z_{k}$. The random variables $w_{k}$ and $v_{k}$ are the process and measurement noise respectively and are assumed to be independent, white, and with normal probability distributions

$$
\begin{aligned}
& p(w) \sim N(0, Q), \\
& p(v) \sim N(0, R),
\end{aligned}
$$

where $Q$ is the process noise covariance and $R$ is the measurement noise covariance.

The kalman filter estimates the process state at some time and then obtains corrections from measurements. The Kalman filter consists of five equations to solve above problem. The equations for the Kalman filter are categorized to two groups which are time update equations and measurement update equations respectively. The time update equations can also be thought of as predictor equations and are listed as follows. 


$$
\begin{gathered}
\hat{x}_{k}^{-}=A \hat{x}_{k-1}, \\
P_{k}^{-}=A P_{k-1} A^{T}+Q,
\end{gathered}
$$

where $\hat{x}_{k}^{-}$is a priori state estimate at step $k$ given knowledge of the process prior to step $k . \hat{x}_{k-1}$ is a posteriori estimate which was updated in the measurement update equations. $P_{k}^{-}$is a priori estimate error covariance. $P_{k-1}$ is a posteriori estimate error covariance which was updated in the measurement update equations.

The measurement update equations can be thought of as corrector equations and are listed as follows.

$$
\begin{gathered}
K_{k}=P_{k}^{-} B^{T}\left(B P_{k}^{-} B^{T}+R\right)^{-1}, \\
\hat{x}_{k}=\hat{x}_{k}^{-}+K_{k}\left(z_{k}-B \hat{x}_{k}^{-}\right), \\
P_{k}=\left(I-K_{k} B\right) P_{k}^{-},
\end{gathered}
$$

where $K_{k}$ is the Kalman gain.

Moreover, $z_{k}$ is known from observing a system. $A, B, Q$ and $R$ are also known parameters while designing a system. The initial values of $\hat{x}_{k-1}$ and $P_{k-1}$ are must determined before running Kalman filter. About the guideline for determining the initial values can be found in 14 .

Since the measurement process of the MBAC-AEBE attempts to estimate the mean and variance of $y(n)$, the state for our system is defined as $x_{k}=\left[M_{k}, V_{k}\right]$, where $M_{k}$ and $V_{k}$ are the mean and variance of $y(n)$ at the $k$-th step. If there is no any traffic entering or leaving the system, the state does not change from step to step so the $A$ in the process equation (4) is set to identity matrix $(I)$. The process equation of our system is:

$$
x_{k}=x_{k-1}+w_{k-1} .
$$

And our noisy measurement is of the state directly so the $B$ in the measurement equation (5) is also set to $I$. So the measurement equation of our system is:

$$
z_{k}=x_{k}+v_{k},
$$

where $z_{k}$ is defined as $z_{k}=\left[\bar{X}_{k}, S_{k}^{2}\right] . \bar{X}_{k}$ is the sample mean of $y(n)$ at the $k$-th step and can be obtained from calculating $(2) . S_{k}^{2}$ is the sample variance of $y(n)$ at the $k$-th step. One way to obtain is to calculate (3). Since $M_{k}$ will adaptively approximate to the mean of the system, we find that the better way to calculate $S_{k}^{2}$ is:

$$
S_{k}^{2}=\frac{\sum_{i=1}^{n}\left(y_{i}-M_{k-1}\right)}{n-1} .
$$


Then, the state $x_{k}$ of our system can be estimated by calculating the equations from (8) to (12) at each step. The process noise covariance $Q$ is set to zero-matrix because we fully "trust" that the state does not change from step to step. The measurement noise covariance $R$ is usually measured prior to operation of the filter. From our experiences, we set $R=\left[\begin{array}{ll}0.1 & 0.1\end{array}\right]^{T} \cdot\left[\begin{array}{lll}0.1 & 0.1\end{array}\right]$. However, the value of $R$ is not critical while $Q$ is set to zero-matrix. The initial value of $\hat{x}_{k-1}$ called $\hat{x}_{0}$ is set to $\left[\begin{array}{ll}0 & 0\end{array}\right]^{T}$ because we observe nothing from $y(n)$ before starting. Furthermore, we choose the initial value of $P_{k-1}$ called $P_{0}$ to be $\left[\begin{array}{ll}1 & 1\end{array}\right]^{T} \cdot\left[\begin{array}{ll}1 & 1\end{array}\right]$. In fact, we can choose almost $P_{0} \neq O$ and the filter will eventually converge.

The values of $M_{k}$ and $V_{k}$ are used to calculate aggregate effective bandwidth when a new traffic requests a connection. The admission decision based on the aggregate effective bandwidth will be discussed in next section. The measurement process continues to run without any change if the new request is rejected. On the contrary, the values of $P_{k-1}, \bar{X}_{k}$ and $S_{k}^{2}$ should be reset when a new request is accepted or an existing traffic is finished.

Since the mean and variance of $y(n)$ will change after a new request is accepted or an existing traffic is finished. We must make $x_{k}$ to approximate the new state (i.e. new mean and new variance). Thus, $P_{k-1}$ is reset as the value of $P_{0}$. This makes the filter does not believe $x_{k}$ is true enough and make $x_{k}$ to approximate the new state. The values of $\bar{X}_{k}$ and $S_{k}^{2}$ should be calculated without consider historical aggregate GoP sizes before the $k$-th step.

\subsection{Admission Decision Using Aggregate Effective Bandwidth}

For the admission decision of the MBAC-AEBE, we consider a model that a PNC has accepted some connections and the capacity is $C$. We assume that the current aggregate bandwidth is $E$. The aggregate GoPs $x(n)$ demands a loss probability no larger than $\epsilon$ which is as follows.

$$
\operatorname{Pr}\left\{\bar{X}_{p}>E\right\}<\epsilon,
$$

where $\bar{X}_{p}=1 / p[x(1)+\ldots+x(p)]$. The MBAC-AEBE tries to find the value of $E$ while given the value of $\epsilon$ and adopts $E$ for admission decision when there is a new request with peak rate $R$. If $E+R<C$, the connection is accepted; otherwise it is rejected.

If $x(n)$ is a Gaussian process, $\bar{X}_{p}$ is also a Gaussian random variable. Furthermore, from the Central Limit Theorem, $\bar{X}_{p}$ approaches to be a Gaussian random variable for $p$ is large enough $(p \geq 30)$ even that $x(n)$ is not a Gaussian process. However, it is difficult to find $E$ accurately by solving (16) if $x(n)$ is non-Gaussian especially for $p$ is small.

Since we have known that $x(n)$ is lognormal distributed, $\ln \left(\bar{X}_{p}\right)$ is exactly a Gaussian random variable. The assumption of a Gaussian distribution allows us to adopt standard approximations to estimate tail probability of the distribution. Then, we can estimate $E$ by solving

$$
\operatorname{Pr}\left\{\ln \left(\bar{X}_{p}\right)>\ln (E)\right\}<\epsilon .
$$


For solving (17), the mean $\hat{\mu}_{l n \bar{X}}$ and variance $\hat{\sigma}_{\ln \bar{X}}^{2}$ of $\ln \left(\bar{X}_{p}\right)$ must be known. From the measurement process of the MBAC-AEBE, the mean $M_{k}$ and variance $V_{k}$ of $y(n)=\ln (x(n))$ have been known. Then, the mean $\hat{\mu}_{x}$ and variance $\hat{\sigma}_{x}^{2}$ of $x(n)$ can be calculated by Fenton's approach [15] as follows:

$$
\begin{gathered}
\hat{\mu}_{x}=\exp \left(M_{k}+\frac{V_{k}}{2}\right), \\
\hat{\sigma}_{x}^{2}=\exp \left(2 M_{k}+V_{k}\right)\left\{\exp \left(V_{k}\right)-1\right\} .
\end{gathered}
$$

Moreover, it is known that the mean $\hat{\mu}_{\bar{X}}$ of $\bar{X}_{p}$ is $\hat{\mu}_{x}$ and the variance $\hat{\sigma}_{\bar{X}_{p}}^{2}$ of $\bar{X}_{p}$ is $\hat{\sigma}_{x}^{2} / p$. The $\hat{\mu}_{\ln \bar{X}}$ and $\hat{\sigma}_{\ln \bar{X}}^{2}$ can be obtained by calculating as follows [16]:

$$
\begin{gathered}
\hat{\sigma}_{\ln \bar{X}}^{2}=\ln \left(\frac{\hat{\sigma}_{\bar{X}}^{2}}{\hat{\mu}_{\bar{X}}^{2}}+1\right), \\
\hat{\mu}_{\ln \bar{X}}^{2}=\ln \left(\hat{\mu}_{\bar{X}}\right)-\frac{\hat{\sigma}_{\ln \bar{X}}^{2}}{2} .
\end{gathered}
$$

Finally, the approximation 17 of $E$ is given by:

$$
E \simeq \exp \left(\hat{\mu}_{\ln \bar{X}}+\alpha^{\prime} \sigma_{\ln \bar{X}}\right), \text { with } \alpha^{\prime}=\sqrt{-2 \ln (\epsilon)-\ln (2 \pi)} .
$$

In brief, it needs to calculate (18)-(22) for the admission decision. Moreover, the value of $p$ in (16) can be non-integer and can be controlled to handle different level of burstness of aggregate traffic. The value of $p$ can be decreased for handling higher level of burstness. The admission decision is involved only when a DEV requests a new connection. The computational complexity of the admission decision is not high. Recalling that the measurement process of the MBAC-AEBE based on the linear Kalman filter is also computational efficiently. Therefore, the MBAC-AEBE is suitable for on-line usage.

\section{Simulation Results}

The scenario of our simulation is described as follows. Different numbers of DEVs are required to transmit VBR video traffic to other DEVs within the same piconet. We accept connections of these different numbers of traffic to evaluate the performance of the measurement process of the MBAC-AEBE and the accuracy of the effective bandwidth for the admission decision of the MBAC-AEBE. We assume that the channel is ideal, in order to focus our attention on the performance of the MBAC-AEBE.

The performance of the measurement process of the MBAC-AEBE is evaluated by checking if the estimated $M_{k}$ and $V_{k}$ can approach to the mean $\mu$ and variance $\sigma^{2}$ of logarithmic aggregate GoP sizes. The logarithmic aggregate GoP sizes with length 3333 GoPs are fitted by the Gaussian distribution and the values of its $\mu$ and $\sigma^{2}$ are found as 13.395 and 0.074 respectively. Furthermore, the sample mean and sample variance are calculated by considering previous 30 
aggregate GoP sizes at each step (GoP index). And the sample mean and the sample variance are fed into the measurement equation (14) of our measurement process.

It is shown in Fig. 2 that the value of the $M_{k}$ well approaches to the value of $\mu$, whereas the sample mean varies largely over time. Therefore, using the estimated $M_{k}$ is much better and more stable than using the sample mean for admission decision.

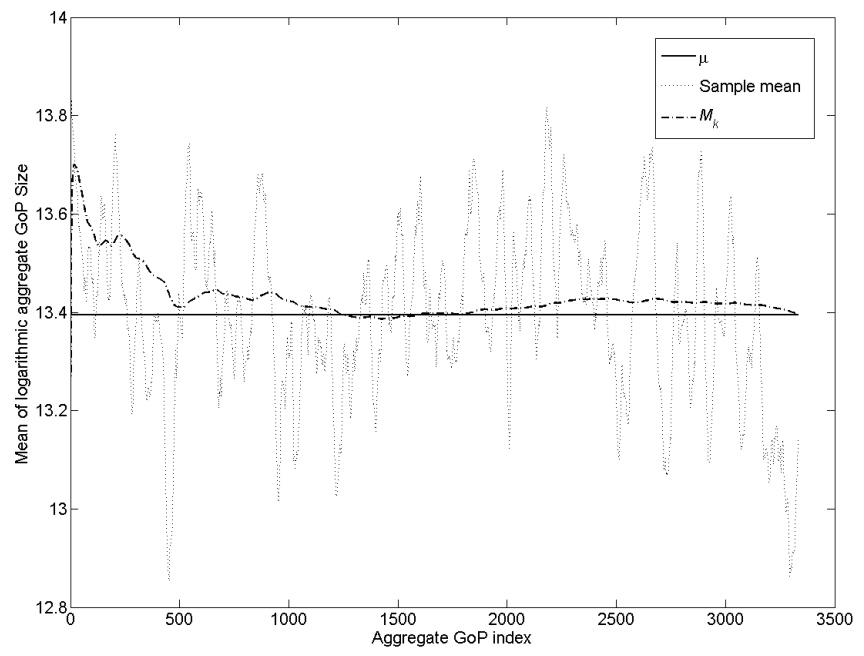

Fig. 2. Performance of the measurement process for estimating the mean $(\mu)$

Since the $M_{k}$ is the good estimation of $\mu$, the MBAC-AEBE adopts (15) to calculate the sample variance and feeds it into the measurement equation. Then, as shown in Fig. 3, the value of the $V_{k}$ also well approaches to the value of $\sigma^{2}$. However, the sample variance calculated by (15) still varies largely over time. Therefore, using the estimated $V_{k}$ is much better and more stable than using the sample variance for admission decision.

Moreover, we evaluate the performance of the admission decision of the MBAC-AEBE. The performance of the admission decision is checked by evaluating the accuracy of the aggregate bandwidth $E$ calculated by (22). The value of loss ratio is more close to the desired QoS (i.e. desired loss ratio) if the value of $E$ is more accurate. The overestimation of the aggregate effective bandwidth may decay channel utilization, whereas the underestimation of the aggregate effective bandwidth may not guarantee the desired QoS. The desired loss ratio is set to $1 \mathrm{e}-4$ in our simulations.

The accuracy of $E$ is evaluated for different numbers of connections which are only 1 connection (Fig. 4), aggregating 12 connections (Fig. 5) and aggregating 18 connections (Fig. 6). It is also compared with the method propose in [17] which assumed that the aggregate traffic is Gaussian distributed. This method is labelled as JSAC91 in our simulations. 


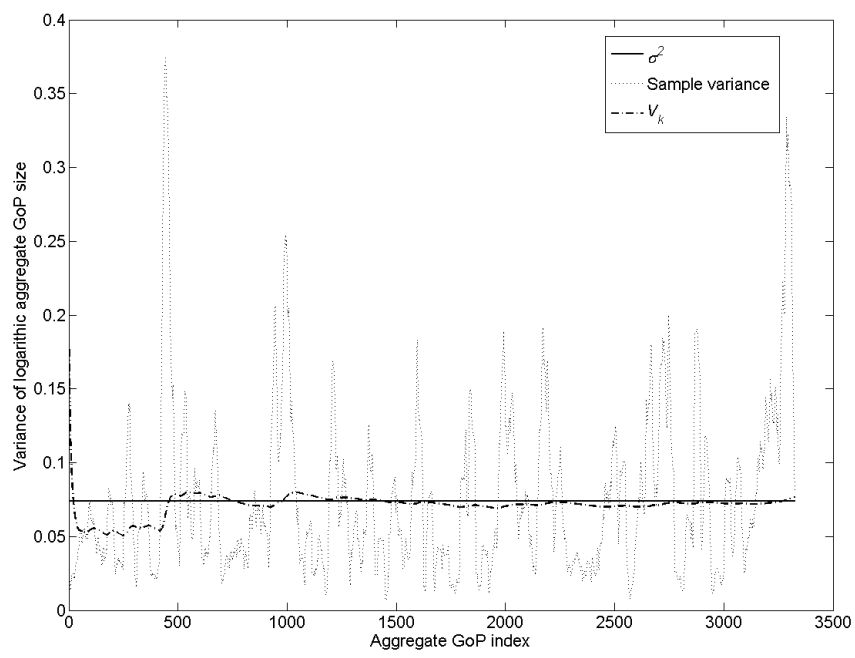

Fig. 3. Performance of the measurement process for estimating the variance $\left(\sigma^{2}\right)$

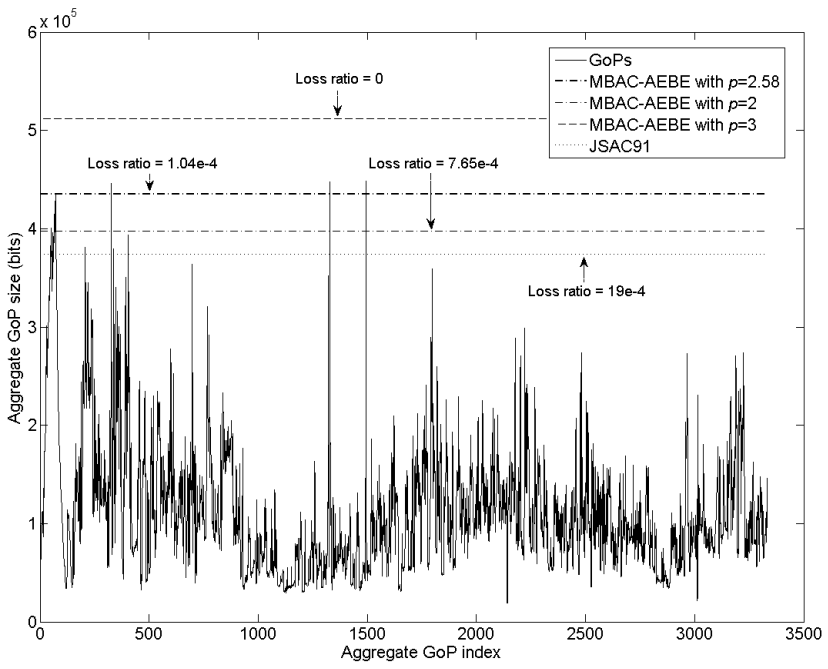

Fig. 4. Accuracy comparison of estimated effective bandwidth for only one connection

Observing the results shown from Fig. 4 to Fig. 6, the accuracy of the JSAC91 is increasing when the number of connections is increasing. This conforms to the Central Limit Theorem because the JSAC91 assumed Gaussian. On the contrary, the MBAC-AEBE is much accurate no matter how many connections are. The resulting loss ratios for the MBAC-AEBE approach to the desired QoS very well such as the MBAC-AEBE with $p=2.58$ in Fig. 4 , the MBAC-AEBE with $p=1.71$ in Fig. 5 and the MBAC-AEBE with $p=1.44$ in Fig. 6 . In practice, the best value of $p$ can be found by using a steepest-descent algorithm [14. 


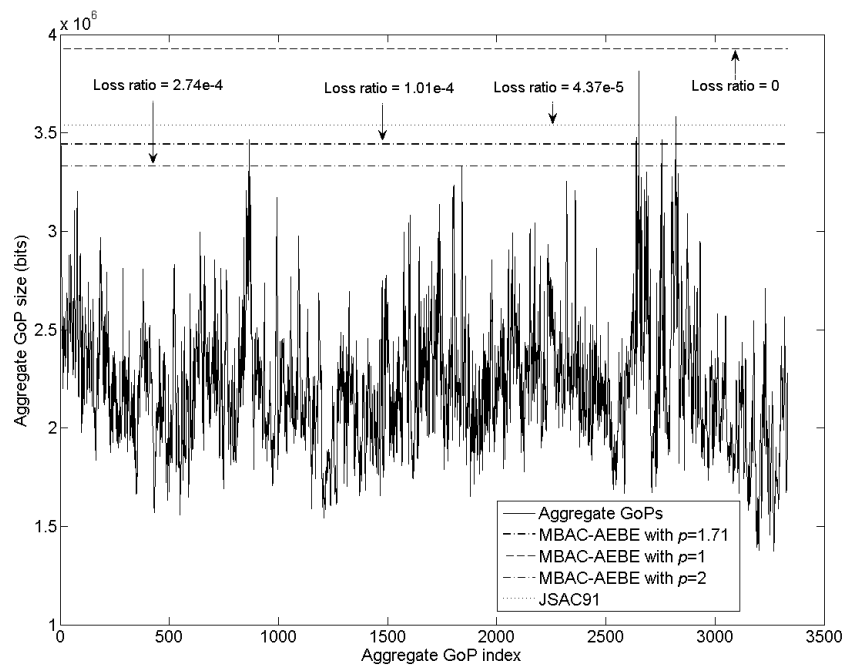

Fig. 5. Accuracy comparison of estimated aggregate effective bandwidth for 12 connections

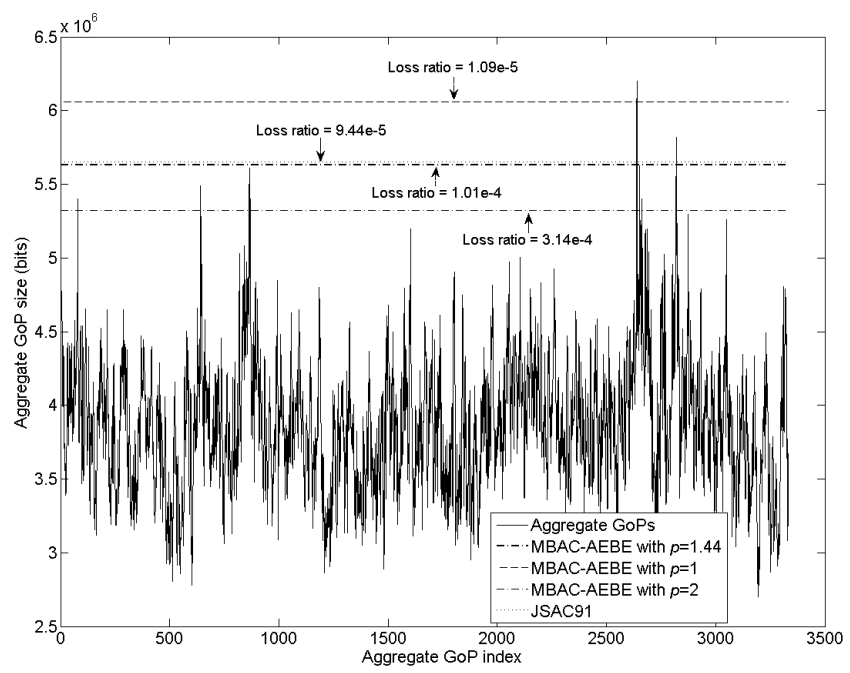

Fig. 6. Accuracy comparison of estimated aggregate effective bandwidth for 18 connections

\section{Conclusion}

This paper proposed an on-line measurement-based admission control scheme called MBAC-AEBE in order to guarantee quality-of-service (QoS) requirements of real-time VBR video traffic. The MBAC-AEBE consisted of two main components which were measurement process and admission decision respectively. 
For on-line usage, the measurement process of the MBAC-AEBE adaptively estimated the mean and variance of logarithmic aggregate GoP sizes in each GoP period by an optimization scheme based on a linear Kalman filter. The Kalman filter could provide an optimal least-square estimate of the system state for a linear system. Then, the estimated mean and variance by measurement process were used to calculate the effective bandwidth for admission decision.

Simulation results showed that the measurement process of the MBAC-AEBE could provide accurate estimations of the mean and the variance of logarithmic aggregate GoP sizes. We also showed that the aggregate effective bandwidth estimated by the MBAC-AEBE could provide loss ratio much approached to the desired QoS. The performance of the admission decision depended on the accuracy of the estimated aggregate effective bandwidth. Moreover, the performance of the admission decision was evaluated for different numbers of connections. It showed that the MBAC-AEBE was much accurate no matter how many connections are.

\section{References}

1. I. Dalqic and F. A. Tobaqi, "Performance evaluation of ATM networks carrying constant and variable bit-rate video traffic," IEEE Journal of Selected Areas in Communications, vol. 15, pp. 1115-1131, 1997.

2. Z. Dziong, M. Juda, and L. G. Mason, "A framework for bandwidth management in ATM networks - aggregate equivalent bandwidth estimation approach," IEEE/ACM Transactions on Networking, vol. 5, no. 1, pp. 134-147, Feb. 1997.

3. K. Shiomoto, S. Chaki, and N. Yamanaka, "A simple bandwidth management strategy based on measurements of instantaneous virtual path utilization in ATM networks," IEEE/ACM Transactions on Networking, vol. 6, no. 5, Oct. 1998.

4. H. Saito and K. Shiomoto, "Dynamic call admission control in ATM networks," Journal of Selected Areas in Communications, vol. 9, no. 7, pp. 982-989, Sept. 1991.

5. T. E. Tedijanto and L. Gun, "Effectiveness of dynamic bandwidth management mechanisms in ATM networks," Proceedings of IEEE INFOCOM '93, pp. 358-367, 1993.

6. K. Shiomoto and S. Chaki, "Adaptive connection admission control using real-time traffic measurements in ATM networks," IEICE Transactions on Communications, vol. E78-B, no. 4, pp. 458-464, Apr. 1995.

7. R. J. Gibbens, F. P. Kelly, and P. B. Key, "A decision-theoretic approach to call admission control in ATM networks," IEEE Journal of Selected Areas in Communications, vol. 13, no. 6, pp. 1101-1114, Aug. 1995.

8. K. Shiomoto, N. Yamanaka and T. Takahashi, "Overview of measurement-based connection admission control methods in ATM networks," IEEE Communications Surveys \& Tutorials, vol. 2, no. 1, pp. 2-13, First Quarter 1999.

9. D. Le Gall, "MPEG: A video compression standard for multimedia applications," Communications of the ACM, vol. 34, pp. 47-58, Apr. 1991.

10. R. E. Kalman, "A new approach to linear filtering and prediction problems," Transaction of the ASME-Journal of Basic Engineering, pp. 35-45, Mar. 1960.

11. IEEE standard 802.15.3: Wireless Medium Access Control (MAC) and Physical Layer (PHY) Specifications for High Rate Wireless Personal Area Networks (WPANs), Inst. Elec. Electron. Eng., New York, USA, 2003. 
12. M. Reisslein et al., "Traffic and Quality Characterization of Scalable Encoded Video: A Large-Scale Trace-Based Study," Arizona State University, Dept. of Elect. Eng., Tech. Rep., Dec. 2003. Video traces available from http://trace.eas.asu.edu.

13. M. Krunz, R. Sass and H. Hughes, "Statistical characteristics and multiplexing of MPEG streams," Proceedings of IEEE INFOCOM '95, vol. 2, pp. 455-462, 1995.

14. S. Haykin, Adaptive Filter Theory. Englewood Cliffs, NJ: Prentice-Hall, 1991.

15. L. F. Fenton, "The sum of lognormal probability distributions in scatter transmission systems," IRE Transactions on Communications Systems, pp 57-67, 1960.

16. K. Nagarajan and G. T. Zhou, "A new resource allocation scheme for VBR video traffic source," Proceedings of 34 th Asilomar Conference on Signals, Systems, and Computers, Pacific Grove, CA, pp. 1245-1249, 2000.

17. R. Guerin, H. Ahmadi, and M. Naghshineh, "Equivalent capacity and its application to bandwidth allocation in high-speed networks," IEEE Journal of Selected Areas in Communications, Vol. 9, pp 968-981, 1991. 